\title{
Phase separation in polymer solutions and gels
}

\author{
R. BANSIL
}

Center for Polymer Studies and Department of Physics, Boston University, Boston, MA 02215, U.S.A.

\begin{abstract}
A brief overview of the kinetics of phase separation in polymer solutions and gels is presented. Small angle light scattering measurements of the time evolution of the structure factor following a quench into the spinodal region for polystyrene cyclohexane solutions show several unusual features as compared to either polymer blends or simple binary liquid mixtures. Nonlinearities appear to be important right from the beginning and the scaling behavior in the intermediate stages indicates the formation of ramified domains. The mobility varies with time, so that the phase separation process slows down as time proceeds. Similar experiments in gelatin solutions in a mixture of water and methanol show the effects of simultaneous occurrence of phase separation and gelation. The most dramatic effect is the pinning of the phase separated morphology at some intermediate stage in the unstable region of the phase diagram upon gelation. The final domain size depends strongly on the quench temperature.
\end{abstract}

On présente un sommaire de la cinétique de séparation des phases dans des solutions de polymères et gels. Diffusion de lumière à petites angles permet de mesurer l'évolution temporelle du facteur de forme qui suit l'abaissement rapide de la température dans la région spinodale. Pour des solutions de polystyrene cyclohexane on trouve quelques caractéristiques inhabituelles comparées aux mélanges de polymères ou aux mixtures simple de liquids binnaires. Les nonlinéarites apparaissent d'etre importantes dès le debut et le comportement de scaling des niveaux intermédiaires indiquent la formation de domains ramifiés. La mobilité varie avec le temps, donc le processus de la séparation des phases ralenti avec le temps. Des experiences similaires avec solutions de gélatine dans une mélange d'eau et methanol montrent les effets de l'apparition simultanée de la séparation des phases et de la gélation. L'effet, le plus dramatique est le blocage de la morphologie des phases separées dans une étape intermédiaire dans la région instable du diagram des phases au moment de la gélation. L'extension du domaine finale depends fortement de la température.

\section{Introduction}

Phase separation and gelation in polymeric systems are of technological as well as fundamental interest. The equilibrium aspects of phase separation phenomena have been extensively studied both in polymer blends and solutions $[1,2]$. The initial process of phase separation usually occurs via the mechanisms of either nucleation or spinodal decomposition $[3,4]$ and is followed by coarsening processes which ultimately result in the formation of two or more distinct phases. 
The kinetics of these processes governs the ultimate morphology, and hence properties, of composite polymer materials. For example the process of immersion precipitation [5] to prepare polymeric membranes involves the simultaneous occurrence of phase separation of the polymer from the solvent component and aggregation or gelation. Thus the process and the ultimate strucrure of the membrane produced is controlled by the kinetics of phase separation and aggregation. Sinilarly in the preparation of polymeric composites it is very common to be in a situation where the two polymeric components may phase separate. Phase separation is commonly used to achieve fractionation. On the other hand, by preventing phase separation it is possible to prepare homogeneous films of two incompatible polymers by dissolving in a common good solvent and then evaporating the solvent. Rapid cooling of concentrated polymeric solutions to dry ice and/or liquid nitrogen temperatures can lead to reversible, physical gelation where microcrystallites or unicrodomains are linked together. An understanding of the kinetics of the different phase separation mechanisms is clearly needed to achieve optimal control in fabrication processes which require one to either prevent phase separation or to promote the formation of phase separated domains. If one knows the time evolution of the domains, then it is feasible to stop the phase siparation process at any desired morphology, for example by inducing a crosslinking reaction to pin the morphology of the domain [6].

The kinetics of spinodal decomposition process in symmetric linear polymer blends has been extensively studied both experimentally [7-12] and theoretically [13-16]. Nucleation in such systems has also been studied [17].

Although phase separation processes in polymer solutions are important technologically and solutions display a wide range of structural and dynamic behavior [18], relatively little work has been done on the kinetics of phase separation in solutions [19-21]. Furthermore, in systems capable of undergoing a sol-gel transition simultaneously with phase separation [22] we have found that the phase separation kinetics is significantly affected by the gelation process and that the system is arrested in a micro-phase separated state [23]. In such systems phase separation can also induce gelation.

\section{General Background on Phase Separation Kinetics}

It is well known that when a binary mixture is quenched to a temperature which lies in the 2phase region of the temperature-composition phase diagram it will eventually phase separate into two distinct phases of composition $C_{p}$ and $C_{r}$ as shown in Fig. 1. Depending on whether the final temperature lies in the metastable or unstable part of the phase diagram the phase separation initially proceeds via the processes of nucleation or spinodal decomposition $[3,4]$, respectively.

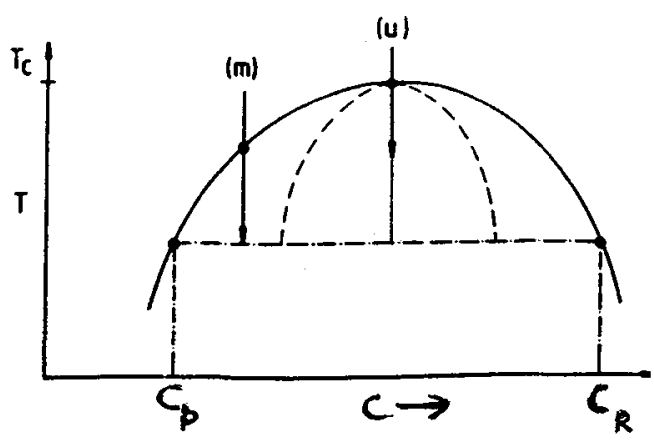

Figure 1. Temperature- composition phase diagram showing the coexistence curve (solid line) and the spinodal curve (dashed). The unstable $(\mathrm{u})$ and metastable $(\mathrm{m})$ regions are labelled. A quench to a temperature $\mathrm{T}$ eventually produces two phases of compositions, $C_{p}$ and $C_{r}$. 
In the process of nucleation the initial mechanism involves the formation of droplets whose composition is that of the minority phase (either $C_{p}$ or $C_{r}$ ) and whose size is greater than some critical size. These droplets grow and eventually coalesce to produce two distinct phases. Spinodal decomposition, on the other hand, involves the formation of a connected, bi-continuous structure and the concentration difference $\delta c$ gradually evolves to the equilibrium composition difference $\left(C_{r}-C_{p}\right)$. The distinction between nucleation and spinodal decomposition is not necessarily sharp; a well defined pseudo-spinodal only exists in systems with long range interactions. At the spinodal temperature the second derivative of the free energy with respect to composition goes to zero and thus the scattered light intensity diverges upon approaching the spinodal temperature from the one phase region. If a sample in the one phase region is suddenly quenched to a temperature below $T_{s}$, the spinodal temperature, concentration fluctuations beyond a critical wavelength begin to grow spontaneously. However, because rapid spatial variations in composition cost interfacial energy while the growth of long length scale fluctuations is limited by diffusion, a characteristic length scale is established in the system. Experimentally this can be seen by the appearance of a. diffraction ring whose diameter is related to the characteristic length scale.

A convenient way of describing this is in terms of the structure factor $S(q, t)$, which is the spatial Fourier transform of the correlation of the concentration fluctuations $\langle\delta c(r, t) \delta c(0, t)\rangle$, and is directly proportional to the scattered intensity measured at a scattering angle $\theta$. Here $q=(4 \pi / \lambda) \sin (\theta / 2)$ denotes the magnitude of the scattering vector, with $\lambda$ the wavelength of the incident beam in the sample. The hallmark of spinodal decomposition is then the appearance of a peak in $S(q, t)$ at $q=q_{m}$ where $q_{m}$ is inversely proportional to the characteristic domain size at which concentration fluctuations are most pronounced. As the process of spinodal decomposition proceeds $q_{m}$ decreases (the domains grow in size) and $S\left(q_{m}, t\right)$ increases (the concentration difference increases) as shown in Fig. 2. Ultimately in spinodal decomposition, as in nucleation, the initial domains thus produced grow (coarsen) and eventually coalesce and segregate to produce two distinct equilibrium phases.
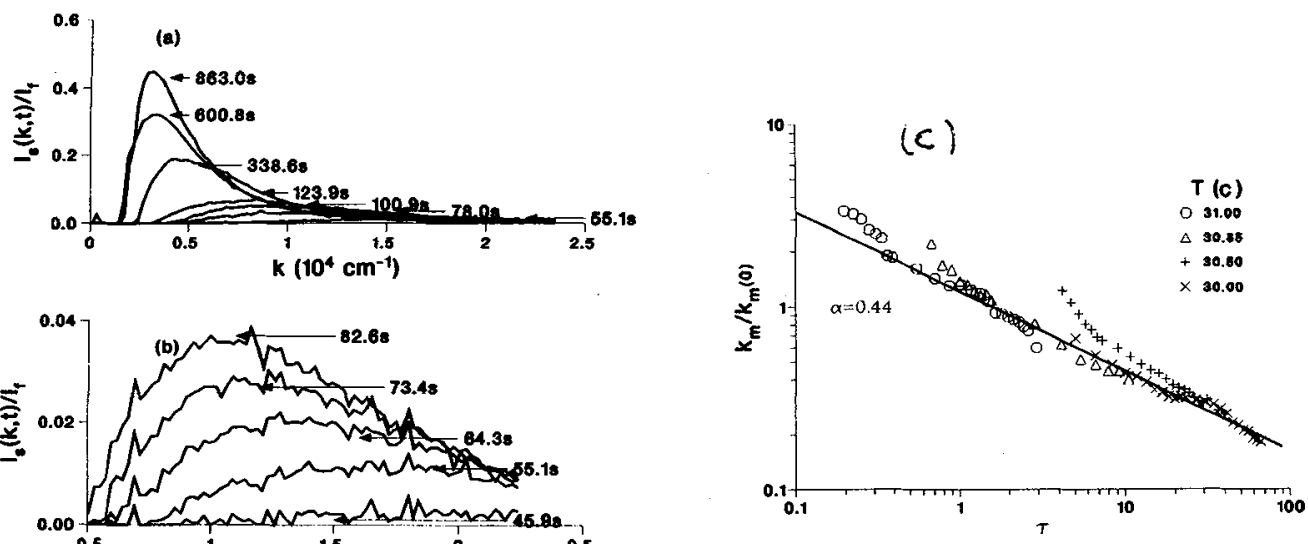

Figure 2. Kinetics of spinodal decomposition measured by SALS on a 3 million molecular weight solution of polystyrene in cyclohexane of critical composition (1\%). (a) Time evolution of the structure factor over the entire duration of the experiment. (b) The early stages of the time evolution showing the peak position shifting from the earliest time. (c) The position of the maximum in the structure factor $q_{m}$ as a function of time showing the unusual rapid growth at "arly times followed by power-law growth. Data for different quenches is shown with the length and time scales normalized by the characteristic inverse correlation length and time constant for each temperature. Details can be found in Lal and Bansil (1991). 
It is common to discuss the process of spinodal decomposition in three stages; early, interinediate and late stages. In the early stages the mean-field linear theory $[15,24,25]$ predicts that concentration fluctuations on the length scale of the correlation length $\xi$ grow exponentially following a temperature or pressure quench with an inverse time constant $\tau(q)=D q^{2}\left(1-q^{2} /\left(2 q_{m}^{2}\right)\right)$, where $D$ is a mobility and $q_{m}=\xi^{-1}$. Short wavelength (i.e., high $q$ ) fluctuations relax exponentially. Several light-scattering studies have found relatively good agreement with linear theory predictions during the early stages of spinodal decomposition $[8,10,26]$.

As concentration differences approach their equilibrium value and domains begin to form, interactions between fluctuations at different wavenumbers can no longer be neglected and the non-linear Cahn Hilliard equation describes the kinetics. While this regime has been extensively observed, there is currently no analytical solution of this nonlinear equation and the only possibilities at present are to either iterate this equation numerically $[27,28]$ or to use the approximate decoupling scheme of Langer et al [29]. Once $\delta c$ reaches the equilibrium concentration difference, the kinetics is dominated by the coarsening of domains [4]. In this late stage regime, power law scaling behavior and structure factor scaling is expected theoretically [4] and has been widely observed $[10,12,30]$. The position of the structure factor peak $q_{m}$ grows as $q_{m} \sim t^{\alpha}$, the maxinum of the peak itself grows as $S\left(q_{m}, t\right) \sim t^{\beta}$ and the structure factor $S(q, t)$ can be scaled into a time-independent function $F\left(q / q_{m}\right)=q_{m}^{3} S(q, t)$. The theoretically predicted values of the exponents $\alpha$ and $\beta$ of $1 / 3$ and 1 , have been observed in computer simulations [27,28] and in a number of light scattering studies of homopolymer blend phase separation kinetics $[10,12,30,31]$. Higher exponents for $\alpha$ have been predicted theoretically and observed when hydrodynamic effects come into play $[32,33]$ or when surface effects due to the presence of walls and finite sample sizes become important $[34,35]$. Wetting effects are probably responsible for growth exponent $\alpha$ $=1.5$ seen in the mixture of two low-molecular weight polymeric liquids [36].

\section{Phase Separation in Polymer Solutions}

The phase behavior of polymer solutions is more complicated than that of blends due to the hig disparity in the size of the two components - the polymer and the solvent molecule-and the unusual effects of polymer-solvent interactions on chain conformation and dynamics. The decreasing interpenetration of polymer chains with decreasing concentration limits the usefulness of mean-field approaches. The Flory interaction parameter $\chi$ must often be taken to be a function of composition in order to fit the asymmetric phase diagrams [37] and the critical exponents which describe the approach to $T_{c}$ are those characteristic of Ising models rather than mean-field values [38-40]. Since the correlation length, the radius of gyration and diffusional dynamics of polymers depends on the concentration, temperature and the $\chi$ parameter, it follows that the phase separation kinetics must be effected as well; the mean-field linear theory approach to the early-stage spinodal decomposition process may not be as successful as in blends. Importance of hydrodynamic effects and the change in diffusional mechanisms as the concentration evolves during phase separation may alter the scaling behavior found in late stages as compared to blends.

(i) Kinetics of nucleation in polymer solutions

Studies of nucleation in polystyrene-cyclohexane (PS-CH) solutions near the coexistence curve [41] revealed a significant difference in the growth rate and size distribution of droplets for two samples whose concentrations lay on either side of the critical point. This work and the recent (xperiments of Nakata and Kawate [42] clearly show the suppression of both the nucleation and droplet growth rate for polymer-poor droplets growing in a polymer-rich solution. Very recent theoretical results [43] confirm our suggestion that these observations are related to the large difference in the viscoelastic properties of the two phases.

(ii) Kinetics of spinodal decomposition in polymer solutions

Early work on spinodal decomposition in polymer solutions [19] focussed only on the growth of the peak intensity with time and analysis it in terms of the linear Cahn-Hilliard model. In our 
recent small angle light scattering (SALS) experiments on PS-CH [20], the time evolution of the entire structure factor was measured for both critical and off-critical quenches for two different molecular weights (cf. Fig. 2). The spinodal curve was determined using static and dynamic light scattering techniques. Several unusual features were seen in the kinetics as compared to either polymer blends or low molecular weight binary fluid mixtures. For example, although the data showed an initial exponential increase in intensity, yet the peak position shifted toward smaller wavenumber even at the earliest times measured. An extensive intermediate regime was observed where the exponents of power-law growth varied continuously (cf. Fig. 2).

We found an unusual scaling behavior of $S(q, t)$ depending on the quench depth. For shallow quenches where most of the data is in the intermediate regime, a time independent scaling function $q_{i n}^{d} S\left(q / q_{m}, t\right)$ could be obtained, although $\mathrm{d} \sim 2$, instead of the usual value of $d=3$ predicted for late stage scaling. We believe that this result may indicate that the interfaces between the forming domains are ramified in this time regime. On the other hand, we observed late-stage scaling of the structure factor with $d=3$ for deep quenches, suggesting that in these cases well defined clomains with sharp interfaces are formed. The late stage scaling function, $F(q) \sim q^{-4}$ which is characteristic of Porod scattering with well formed domains instead of the percolating structure predicted by Furukawa [44] for critical quenches into the spinodal regime. Similar results were obtained by Wiltzius et al [11] in polymer blends. However, for the intermediate stage data the $q$-dependence of the normalized structure factor was also unusual, $F(q) \sim q^{-3.5}$, suggestive of a diffuse (perhaps fractal) interface,

The exponents characterizing the effective power law growth of the characteristic wavenumber, $q_{m}$ also show differences between the intermediate and late stages. At late stages the exponent was close to $1 / 3$ which is in agreement with the results on polymer blends and also with the predictions of droplet growth models $[31,45]$. However in the intermediate stages the exponent varied with quench depth and was close to $1 / 2$. Exponents greater than $1 / 3$ are usually attributed to the effects of hydrodynamic interactions [32] and in normal binary fluid mixtures observed in the late stages of the phase separation process $[3,46,47]$. In contrast, our results suggest that in dilute polymer solutions hydrodynamic interactions may be important in the early stages.

We suggest that in a dilute solution the initial stages of phase separation occur with a Zimmlike diffusion where hydrodynamic interactions are important. As phase separation proceeds the polymer molecules are predominantly in regions of higher concentrations where entanglements cause hydrodynamic screening and thus the late stage growth is characteristic of droplet or clustercluster growth. To further test this hypothesis we examined the growth law exponents in an off-critical high molecular weight PS-CH solution $\left(M_{w}=3 \times 10^{6}, c=7 \%\right.$ where hydrodynamic interactions would be screened right from the beginning. Here we found that the mean-field exponents provide a better fit to the divergence of the correlation length on approaching the spinodal. Results on the time evolution of the structure factor are shown in Fig. 3 for three different quench temperatures. We found that the growth kinetics for the quench to $303.35 \mathrm{~K}$ (Fig. 3c), which is just above the spinodal, was essentially the same as that for the quenches below the spinodal (Fig. 3a, b). Thus there seems to be a gradual transition from the metastable to the unstable region in the vicinity of the spinodal, with behavior on either side of the mean field spinodal line being the same. With this sample we were able to follow the kinetics from early to late stages for the deepest quench $(300.5 \mathrm{~K})$ and as shown in Fig. 3d the inverse of the domain size. $q_{m} \sim t^{-\alpha}$ with $\alpha$ close to 0.25 at early times (characteristic of Langer Bar-on Miller model) followed by $\alpha=1 / 3$ (characteristic of the Lifshitz-Slyozov growth mechanism) and eventually crossing over to $\alpha=1$ in late stages when hydrodynamic effects cannot be neglected.

In order to better understand the morphology of the late-stage domain coarsening process, we used video microscopy to examine the kinetics in real space. For off-critical quenches, as shown in Fig. 4a-f, we can clearly observe the change from a connected (ramified) morphology to isolated compact domains. The effects of the walls of the thin sample cell can also be clearly 

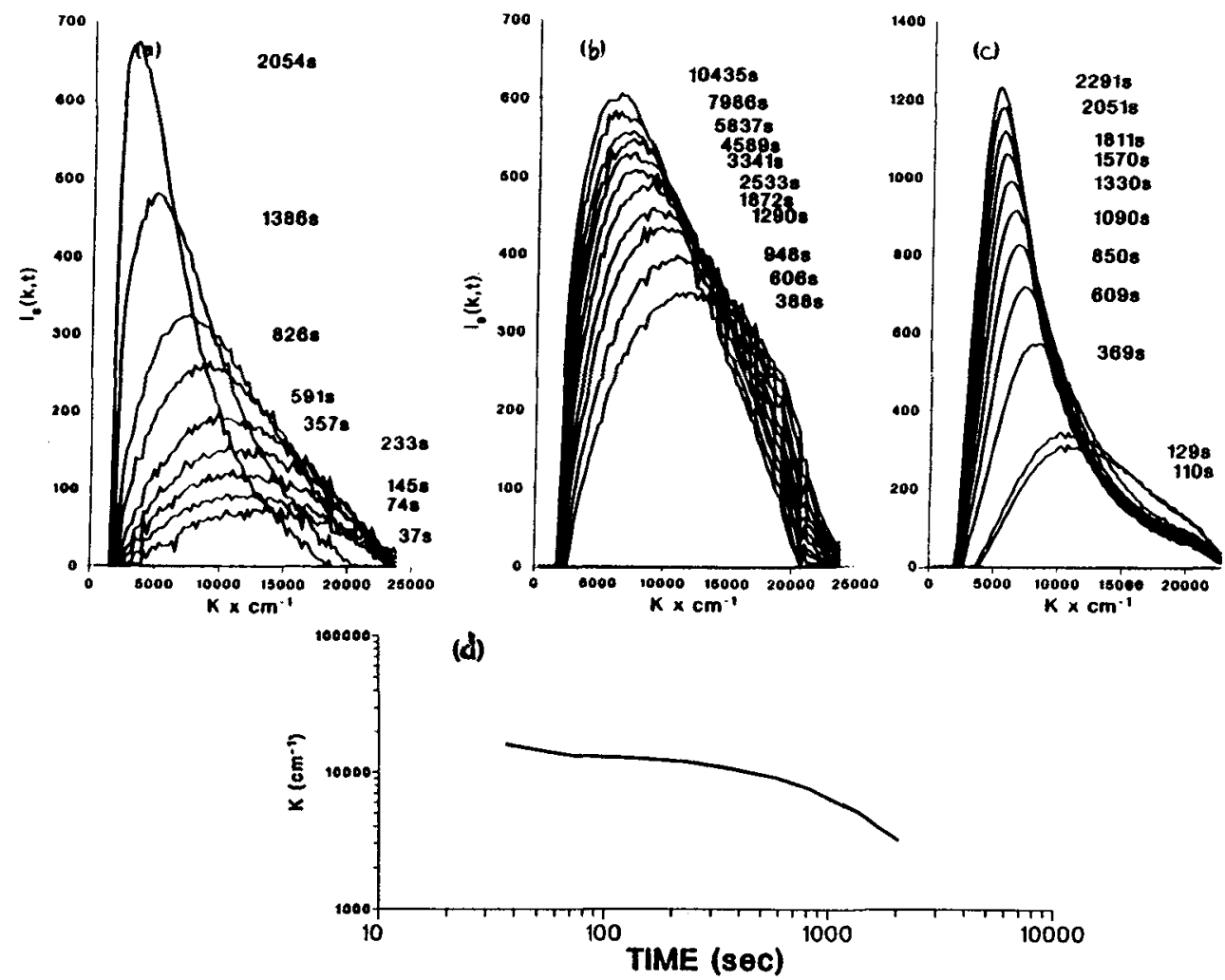

Figure 3 . Time evolution of the scattered intensity following a quench to different temperatures in the two-phase region for an off-critical solution of polystyrene cyclohexane $(7 \%$ solution of $M_{w}=3 \times 10^{6}$ ). The data shown in (a) and (b) are for temperatures below the spinodal, 300.5 and $301.0 \mathrm{~K}$, respectively. (c) Similar results are obtained for a quench to $303.35 \mathrm{~K}$, which is above the spinodal, i.e. in the metastable region. (d) The time-dependence of the peak position $q_{m}$ for the quench to $300.5 \mathrm{~K}$ showing the variation of the power-law growth exponent from $1 / 4$ in the early stages to 1 at late stages.

observed at late stages, where bubbles of diameter equal to the cell thickness $(0.1 \mathrm{~mm})$ form. Faint outlines of these large bubbles appear simultaneously with well defined smaller droplets. The coalescence of smaller droplets is clearly visible. In late stages it appears that the small droplets get incorporated into the pre-existing larger bubbles. When the larger bubbles coalesce the domains become highly ellipsoidal, suggesting that while the in-plane dimension increases, growth in the dimension perpendicular to the surface of the cell is limited to the thickness and this determines the size of the minor axis of the ellipsoid of revolution. Analysis of the characteristic size of the domains shows a growth with an exponent close to $1 / 3$ for the smaller droplets and a higher exponent of $1 / 2$ for the larger bubbles.

\section{Phase Separation in Polymer Gels}

Gelation of polymer solutions involves a connectivity phase transition which causes the viscosity to diverge at the gel point. Since binary phase separation is driven by a diffusive process it is very likely that the onset of gelation would drastically slow down the kinetics of the phase separation process. The question of how crosslinking affects phase separation is particularly important 



Figure 4. A series of time lapse pictures showing the evolution of the structure in a $7 \%$, 3.1 million molecular weight Polystyrene-Cyclohexane solution quenched to $29.3 C$. A connected structure is clearly evident at 10 minutes (a) after the quench. The pinching off of domains becomes more and more distinct as time evolves, as shown in figures b, c, d, e, and $f$ (at times 30 minutes, 1.9 hours, 2.75 hours, 4.33 hours, and 6.2 hours, respectively), with individual bubbles growing to the width of the cell $(0.1 \mathrm{~mm})$ in figure $f$. The length scale for figures a-c is $1 \mathrm{~cm}$ $=0.04 \mathrm{~mm}$ and for figures $\mathrm{d}-\mathrm{f}$ is $1 \mathrm{~cm}=0.06 \mathrm{~mm}$.

for processes where one wishes to control the morphology of a polymeric product by inducing crosslinking during the phase separation process. In these situations the behavior may be quite dependent on the nature of the crosslink, i.e., whether it is a permanent covalent crosslink or a 
temperature reversible weak bond.

Many biopolymers as well as synthetic polymers form temperature reversible, physical gels $[48,49]$. In such systems it is possible to choose a solvent such that the sol-gel transition occurs in the same temperature regime as liquid-liquid phase separation. This leads to a coupling of the two transitions and micro-phase separated gels are produced. Since the sol-gel line depends on concentration, gelation can be induced in the polymer-rich regions formed during the process of phase separation; the occurrence of gelation pins the phase separation process leading to microphase separated regions. This phenomenon is the basic principle underlying the technology of preparing macroporous membranes by immersion precipitation [5].

The equilibrium thermodynamics of micro-phase separation in the synthetic, reversible (i.e., physical) polymer gels has been extensively studied in recent years both theoretically [50,51] and experimentally [22,52-57]. Since the morphology of these gels is kinetically controlled we have recently investigated the kinetics of this coupled phenomenon using gelatin in water + methanol where phase separation and gelation occur in a very convenient temperature range $(20-35 \mathrm{C})$ $[23]$.

Falling ball micro-viscometry was used to determine the kinetics of the sol-gel transition and also to determine the behavior of time to gel, $\tau_{\mathrm{gel}}(T)$, as a function of quench temperature. Static and dynamic light scattering techniques [58] were used to determine the equilibrium phase diagram and spinodal temperature. Small angle light scattering was used to measure the time evolution of the structure-factor following a quench below the spinodal temperature.

We found that the kinetics of phase separation depends very strongly on temperature; and for deep quenches where the rates of phase separation and gelation are comparable, the phase separation process does not go to completion.

For deep quenches after the onset of gelation, $k_{m}$ remains unchanged with time, while the peak intensity exhibits a slow growth. The final domain size, $k_{\text {final }}^{-1}$, at which the phase separating morphology becomes pinned decreases with increasing quench depth (see Fig. 5a,b).

The influence of gelation on phase separation can be seen in optical micrographs which reveal the network structure and well formed domains of high and low contrast corresponding to variation in polymer concentration (Fig. 5c). Since the sample does not phase separate fully into two distinct phases the early stage percolating structure characteristic of the spinodal decomposition process is pinned due to the onset of gelation. This is in stark contrast to the morphology seen in the phase separating polystyrene solutions (Fig. 4), where isolated domains develop from the initial percolating structure and these droplets ultimately coalesce to form two macroscopic phases.

In an effort to determine whether spinodal decomposition plays a role in the technological process of immersion precipitation [5] we prepared such a membrane by immersing a $16 \%$ aqueous solution of gelatin into a methanol bath. The resulting membrane was removed from the methanol bath after 8 minutes and quenched in a liquid-nitrogen bath to preserve the structure. The membranes were dried under vacuum and then sputtered with a $200 \AA$ layer of gold. The morphology of the dried membranes was examined with an Etec Autoscan Scanning Electron Microscope. The electron micrographs clearly reveal a connected structure with well defined micron-sized pores in a network of gelatin fibers (see Fig. 5d). By comparing with Fig. 5c, it is clear that such a structure can result by spinodal decomposition coupled to a gelation process. Similar structure of gelatin networks has been observed at higher magnification in electron micrographs of gelatin filuns cast from aqueous solutions [59].

\section{Conclusion}

While studies of spinodal decomposition kinetics in polymer solutions are still very recent, the brief overview presented here shows that differences with kinetics of both symmetric polymer blends and low molecular weight binary fluid mixtures are becoming apparent. Nonlinearities appear to be more important in the early stages in the solutions and, in the intermediate stages, 

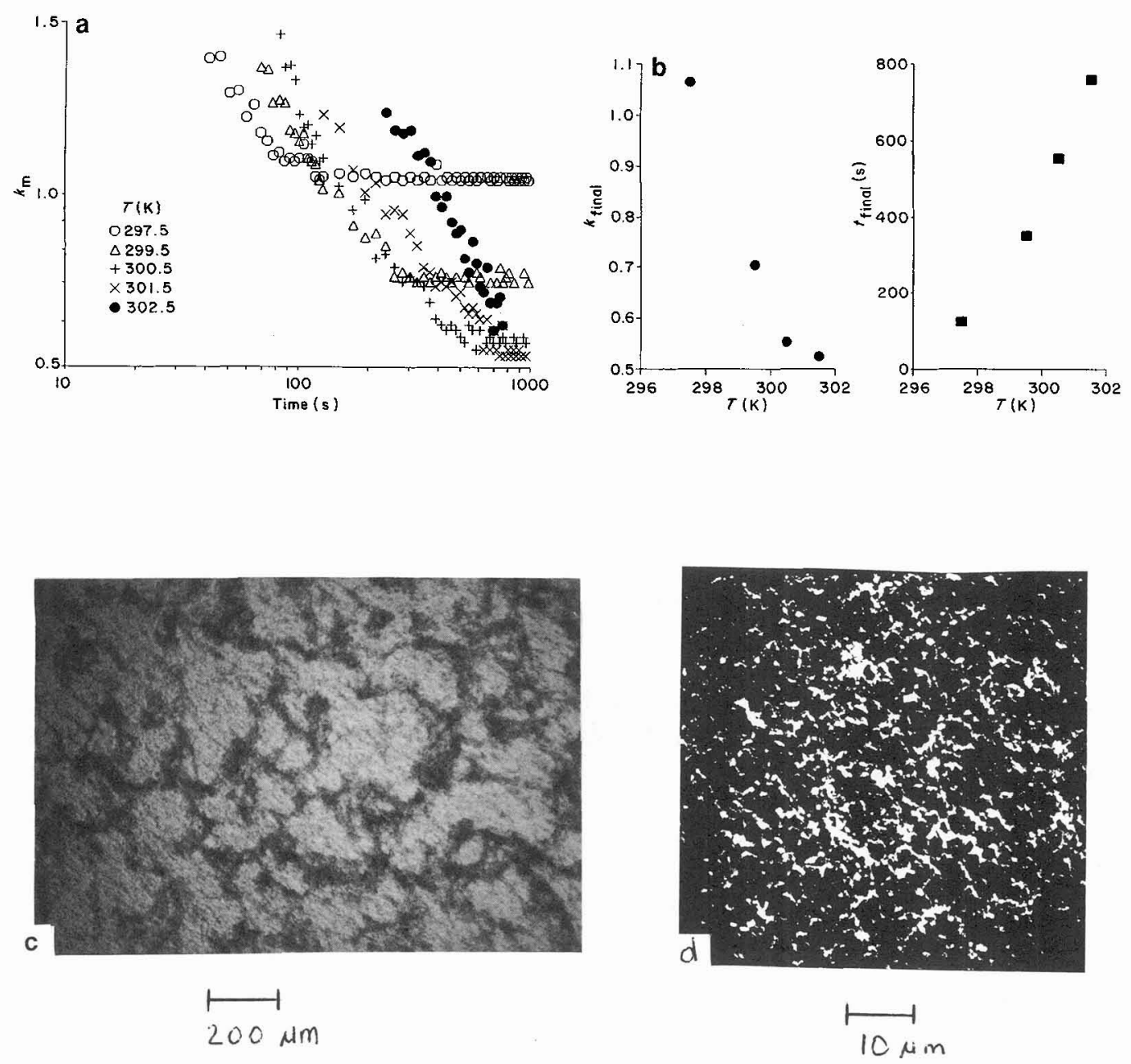

Figure 5. Kinetics of spinodal decomposition in a 7\% solution of gelatin in water-methanol mixture quenched to different temperatures below the spinodal. (a) Time dependence of the maximum in the structure factor, $q_{m}$ (units of $\mu m^{-1}$ ). (b) Temperature dependence of the maximum, $q_{f}$ and time, $t_{f}$ at which domain growth stops due to gelation. (c) Optical micrograph with a $40 \mathrm{X}$ magnification showing the morphology of the phase separated domains 24 hours after the sample was quenched to $22 \mathrm{C}$. The connected morphology characteristic of spinodal decomposition is preserved due to gelation, leaving the sample in a micro-phase separated state. (d) A scanning electron micrograph of an immersion precipitate membrane made from gelatin showing a highly connected porous structure with gelatin fibers and micron sized holes.

our results indicate that the domains are ramified. The mobility varies with time, so that the process of phase separation slows down as time proceeds. Some of the effects are related to the asymmetry of the phase diagram and the very different rheological properties of the two phases. The unusual features of the kinetics in a dilute solution are probably related to the crossover from Zimm dynamics in the dilute solution to Rouse and reptation behavior as the phase separation 
process leads to the formation of domains of high polymer concentration. It is clear that a study of polymer mixtures where the molecular weight ratio of the components is systematically tuned should provide insight into the crossover from mean-field like behavior of the symmetric polymer blend to nonlinear behavior characteristic of polymer solutions.

In solutions capable of gelling the phase separation process is arrested at some intermediate state, pinning the morphology of the sample. The strong temperature dependence of this phenomenon suggests that by controlling the temperature it should be possible to control the unorphology from either isolated domains to connected domains.

The results also show that the linear theory does not describe the experiments in polymer solutions. At present we are testing the applicability of non-linear approaches to describe the spinodal decomposition kinetics in polymer solutions and gels. Specifically we are considering the Langer, Bar-on, Miller (LBM) decoupling scheme [29] with free energy functions appropriate for polymer solutions to see if the non-linearities included in this model are sufficient to explain the experimentally observed non-linear effects at early and intermediate times.

Another solution is to use numerical integration [28] to solve the non-linear Cahn Hilliard equation. We have used this approach in investigating the simultaneous gelation and phase separation kinetics [60]. Results for 2-dimensional calculations show all the features observed in the experiments on gelatin, namely the pinning of structure factor and the pronounced temperature dependence of the final wavevector at which domain growth stops.

\section{ACKNOWLEDGEMENTS}

The author wishes to thank Dr. Preben Alstrom, Mr. James Douglas Bradley, Dr. Bruce Carvalho, Dr. Patrick Gallagher, Dr. Sharon Glotzer, Dr. Jyotsana Lal, Dr. Francesco Sciortino and Dr. H. E. Stanley for their collaboration in this research. This research has been supported by grants from NSF and British Petroleum.

\section{References}

[1] Flory, P. J., Principles of Polymer Chemistry (Cornell Univ. Press, Ithaca, 1953).

[2] Paul, D. R. and Newman, S. (eds), Polymer Blends Vols. I and II (Academic Press, New York, 1978).

[3] Goldburg, W. E., in Scattering Techniques Applied to Supramolecular and Nonequilibrium Systems, eds. S. H. Chen, B. Chu, and R. Nossal (Plenum Press, New York, 1980).

[4] Gunton, J. D., San Miguel, M., and Sahni, P. S., in Phase Transitions and Critical Phenomena Vol. 8, eds. Domb and Lebowitz, 1983.

[5] Reuvers, A. J., Altena, F. W., and Smolders, C. A., Jour. Pol. Sci. 24 (1986) 793.

[6] Hashimoto, T., Takenaka, M., and Jinnai, H., Polymer Communications 30 (1989) 177.

(7) Snyder, H. L. and Meakin, P., J. Chem. Phys. 79 (1983) 5588.

[8] Okada, M. and Han, C. C., J. Chem. Phys. 85 (1986) 5317.

[9] Nishi, T., Wang, T. T., and Kwei, T. K., Macromolecules 8 (1975) 227.

[10] Hashimoto, T., in Phase Transitions 12 (1988) 47, and references therein.

[11] Wiltzius, P., Bates, F. S., and Heffner, W. R., Phys. Rev. Lett. 60 (1988) 1538.

[12] Bates, F. S, and Wiltzius, P. J., Chem Phys 91 (1989) 3258.

[13] de Gennes, P.-G., J. Chem. Phys. 72 (1980) 4756.

[14] Pincus, P., J. Chem. Phys. 75 (1981) 1996.

[15] Binder, K., J. Chem. Phys. 79 (1983) 6385.

[16] Binder, K., in Materials Science and Technology: Phase Transformation in Materials ed. P. Haasen [VCH Verlagsgesellschaft, Germany] 5 (1990) 1.

[17] Cummings, A. and Wiltzius, P., Phys. Rev. Lett. 65 (1990) 863.

[18] des Cloizeaux, J. and Jannink, G., Polymers in Solution (Clarendon Press, Oxford, 1990).

[19] van Aaarsten, J. J. and Smolders, C. A., Euro. Poly. J. 6 (1970) 1105. 
[20] Lal, J. and Bansil, R., Macromolecules 24 (1991) 290.

[21] Kubota, K. and Kuwahara, N., Phys. Rev, Lett. 68 (1992) 197.

[22] Tanaka, T., Swislow, G., and Ohmine, I., Phys. Rev. Lett. 42 (1979) 1556.

[23] Bansil, R., Lal, J., and Carvalho, B. L., Polymer 33 (1992) 2961

[24] Cahn, J. W. and Hilliard, J. E., J. Chem. Phys. 28 (1958) 258.

[25] Cook, H. E., Acta Metall 18 (1970) 297.

[26] Sato, T. and Han, C. C., J. Chem. Phys. 88 (1988) 2057.

[27] Chakrabarti, A., Toral, R., and Gunton, J. D., Phys. Rev. Lett. 63 (1989) 2661.

[28] Chakrabarti, A., Toral, R., Gunton, J. D., and Muthukumar, M., J. Chem. Phys. 92 (1990) 6899.

[29] Langer, J. S., Bar-on, M., and Miller, H., Phys. Rev. A 11 (1975) 1417.

[30] Tomlins, P. E. and Higgins, J. S., J. Chem. Phys. 90 (1989) 6691.

[31] Nose, T., in Space-Time Organization in Macromolecular Fluids 51, eds. F. Tanaka, M. Doi and T. Ohta (Springer-Verlag, New York, 1989), p. 40.

[32] Kawasaki, K., Progr. Theor. Phys. 59 (1977) 362.

[33] Siggia, E. D., Phys. Rev. A 20 (1979) 595.

[34] Jones, R. A. L., Norton, L. J., Kramer, E. J., Bates, F. S., and Wiltzius, P., Phys. Rev. Lett. 66 (1991) 1326.

[35] Binder, K., Proc of Internat"l Work on "Systems with a Liquid Miscibility Gap" Bad Honel (1992).

[36] Wiltzius, P. and Cumming, A., Phys. Rev, Lett. 66 (1991) 3000.

[37] Koningsveld, R., Keintjens, L. A., and Shultz, A. R., J. Poly. Sci. 8 (1970) 1261.

[38] Chu, B., Schoenes, F. J., and Fisher, M. E., Phys. Rev. 85 (1969) 219.

[39] Kojima, J., Kuwahara, N., and Kaneko, M., J. Chem. Phys. 63 (1975) 333.

[40] Perzynski, R., Delsanti, M., and Adam, M., J. Phys. 48 (1987) 115.

[41] Krishnamurthy, S. and Bansil, R., Phys. Rev. Lett. 50 (1983) 2010.

[42] Nakata, M. and Kawate, K., Phys. Rev. Lett. 68 (1992) 2176.

[43] Onuki, A., "Viscoelastic Effect on Nucleation in Semidilute Polymer Solutions," 1992 preprint.

[14] Furukawa, H., Adv. Phys. 34 (1985) 703.

[45] Lifshitz, I. and Slyozov, V., J. Phys. Chem. Solids 19 (1961) 35.

[46] Wong, N. C. and Knobler, C. M., J. Chem. Phys. 69 (1978) 725.

[47] Guenoun, P., Gastaud, R., Perrot, F., and Beysens, D., Phys. Rev. A 36 (1987) 4876.

[48] Russo, P. S. (ed.), Reversible Polymeric Gels (ACS Symposium Series, Washington DC, 1987).

[49] Mandelkern, L., Edwards, C. O., Domszy, R. C., and Davidson, M. W., Microdomains in Polymer Solutions, ed. P. Dubin (Plenum, New York, 1985), p. 121.

[50] Coniglio, A., Stanley, H. E., and Klein, W., Phys. Rev. B 25 (1982) 6805.

[51] Tanaka, F., Macromlecules 23 (1990) 3790.

[52] Wellinghoff, X., Shaw, J., and Baer, E., Macromolecules 12 (1979) 932.

[j3] Tan, H.-M., Moet, A., Hiltner, A., and Baer, E., Macromolecules 16 (1983) 28.

[54] Domszy, R. C., Alamo, R., Edwards, W., and Mandelkern, L., Macromolecules 19 (1986) 316.

[55] Lee, J., Kim, H., and Yu, H., Macromolecules 21 (1988) 858.

[56] Guenet, J.-M. and McKenna, G. B., Macromolecules 21 (1988) 1752.

[57] Hikmet, R. M., Callister, S., and Keller, A., Polymer 29 (1988) 1378.

[58] Chu, B., Laser Light Scattering: Basic Principles and Practice (Academic Press, New York, 1991).

[59] Bradley, J. D., in Proc. of the Sixth Nat'l Conf. on Undergraduate Research (UNCA, Ashville, 1992).

[60] Sciortino, F., Bansil, R., Alstrøm, P., and Stanley, H. E., Phys. Rev. E (1993) in press. 